Proceedings

\title{
Quasi-Static and Dynamic Testing of Annealed Float Glass ${ }^{\dagger}$
}

\author{
Karoline Osnes 1,2, , Odd Sture Hopperstad 1,2 and Tore Børvik 1,2 \\ 1 Structural Impact Laboratory (SIMLab), Department of Structural Engineering, Faculty of Engineering, \\ Norwegian University of Science and Technology (NTNU), NO-7491 Trondheim, Norway; \\ odd.hopperstad@ntnu.no (O.S.H.); tore.borvik@ntnu.no (T.B.) \\ 2 Centre for Advanced Structural Analysis (CASA), NTNU, NO-7491 Trondheim, Norway \\ * Correspondence: karoline.osnes@ntnu.no \\ + Presented at the 18th International Conference on Experimental Mechanics (ICEM18), Brussels, Belgium, \\ 1-5 July 2018.
}

Published: 29 June 2018

\begin{abstract}
Three different experimental set-ups were employed in the investigation of the fracture strength of annealed float glass. These included four-point bending tests on three different specimen sizes, and quasi-static and dynamic pressure tests. The latter tests were conducted in a shock tube, i.e., a device that produces pressure loadings similar to that from a far-field blast event. The experimental work demonstrated that the fracture strength of glass varies largely within the same test set-up. It also revealed that the fracture strength and its scatter are dependent on the size of the specimen, the boundary conditions and the loading situation. By employing a newly proposed strength prediction model, which relies on the physical fracture behaviour of glass, the trends observed in the experiments were correctly recreated.
\end{abstract}

Keywords: float glass; brittle fracture; stochastic fracture; blast load; shock tube

\section{Introduction}

In recent years, there has been a large increase in the use of glazed areas in buildings. This includes glass in large panels, roofs and beams, in addition to entire facades. As a result, the glass is required to act as a structural component that needs to carry considerable loads. Additionally, due to the terrorist threats in today's society, glass structures must often be designed to withstand extreme loadings such as blast pressures from explosions. In the event of such an attack, the most frequent injuries are due to accelerated fragments of failed glass components [1]. This increases the complexity of the design process, and calls for a better understanding of glass' load-carrying capacity. It further requests the development of numerical tools and models capable of predicting fracture and crack propagation in glass.

It is widely known that the fracture strength of glass is stochastic in nature [2], and is due to the presence of micro-structural surface flaws. These flaws cause glass plates to fail primarily in tension, with opening (mode I loading) and growth of the flaws [3]. The identification of the fracture strength of glass is not straightforward, and depends on the combination of the flaw properties and the applied stress. Consequently, the glass strength is dependent on the geometry of the structural component, the boundary condition and the loading situation. The fracture initiation may also not necessarily occur at the point of maximum stress. The complexity of the design process is increased when including effects of strain rate, as present in rapid loading scenarios. This is because flaws need time to grow [4], but this effect is challenging to quantify. There is naturally a great need for numerical methods capable of predicting the fracture behaviour of glass. However, it is also necessary to 
perform experiments for use in validation of such models. Additionally, needless to say, these experiments need to be done in a precise manner with the use of accurate measuring techniques.

In this work, three different experimental set-ups are used to investigate the fracture strength of annealed float glass depending on specimen geometry, boundary condition and loading situation. This includes quasi-static four-point bending tests on three different test specimens of a relatively small size, and uniform quasi-static and dynamic pressure tests on larger glass plates. The dynamic pressure tests involve subjecting the glass plates to a blast pressure by use of the SIMLab Shock Tube Facility [5], while the quasi-static pressure tests employ a slowly increasing water pressure.

The results from the experiments will be used to validate a strength prediction model of glass [6], which is based on the work by Yankelevsky [7]. This stochastic model aims at predicting the glass strength without the need for material tests.

\section{Material and Methods}

\subsection{Glass Material}

All specimens used for the experimental work in this study are made up by clear annealed sodalime silica glass. Glass is generally a brittle material and behaves linear-elastically to the point of fracture. Furthermore, fracture in glass is a highly stochastic process, to which microscopic surface flaws are responsible. When these flaws are subjected to tensile stresses, they will open and, if the stresses are of a critical value, grow into larger cracks in an unstable manner. This will normally lead to glass failure, as the chance of crack arrest for glass is relatively small. The fracture strength (or the onset of unstable flaw growth) of glass can be determined by linear fracture mechanics, i.e.,

$$
K_{I}=K_{I C}, \quad K_{I}=Y \sigma \sqrt{\pi a}
$$

Here, $K_{I}$ is the stress intensity factor for mode I loading (opening of a crack), $K_{I C}$ is the fracture toughness, $\sigma$ is the remote stress normal to the flaw, $Y$ is a geometric factor, and $a$ is the flaw depth. $Y$ is further dependent on the flaw shape $a / c$, where $c$ is the length of the flaw [8].

\subsection{Digital Image Correlation}

In the tests on the larger glass plates, a three-dimensional point-tracking procedure was employed to obtain the deformation of the glass (and the velocity of free-flying glass fragments) during the tests. White circles with a central black dot (optical targets) were painted on the glass, while the in-house Digital Image Correlation (DIC) code eCorr [9] was used to track their displacements. The procedure has earlier been validated against laser measurements [6].

\subsection{SIMLab Shock Tube Facility}

The SIMLab Shock Tube Facility (SSTF) was employed to subject glass plates to a pressure loading close to that from a far-field explosion. This is done by a build-up air pressure, which is sent through the shock tube and eventually transforms into a blast wave. When the blast wave reaches the glass specimen, which is placed at the rear end of the shock tube, it is reflected. The reflected pressure represents the loading experienced by the tested specimen. For a more thorough description of the SSTF, and the general experimental set-up, we refer to Aune et al. [5].

\section{Experiments}

\subsection{Four-Point Bending Tests}

Quasi-static four-point bending tests of glass were performed on three different specimen sizes, denoted small $(100 \mathrm{~mm} \times 20 \mathrm{~mm} \times 4 \mathrm{~mm})$, medium $(200 \mathrm{~mm} \times 40 \mathrm{~mm} \times 4 \mathrm{~mm})$ and large $(300 \mathrm{~mm} \times$ $60 \mathrm{~mm} \times 4 \mathrm{~mm}$ ) specimens [6]. The support span was $20 \mathrm{~mm}$ shorter than the length of the specimens, while the loading span was half of the support span. High-strength steel cylinders with a diameter of $6 \mathrm{~mm}$ were used for support and loading application. Specimens failing at the edges were 
disregarded in this study, which led to a total of 20, 21, and 30 (out of 31) valid tests for the small, medium and large specimens, respectively. The applied load at failure is presented in Figure 1. Note that the ordinate, denoted the probability density, refers to a normalized occurrence. This is calculated by $c_{i} /\left(N w_{i}\right)$, where $c_{i}$ is the number of elements in the bin, $N$ is the total number of elements and $w_{i}$ is the width of the bin.

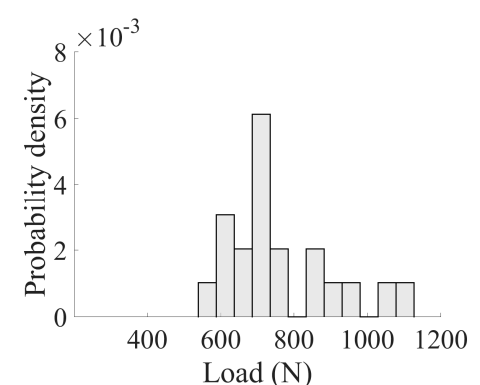

(a)

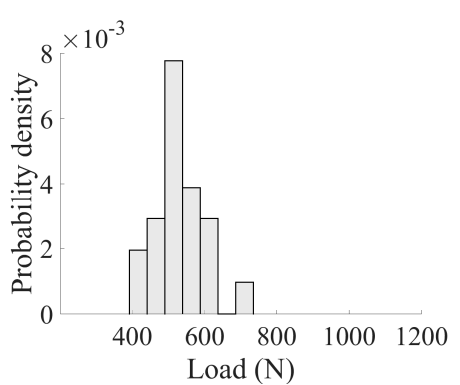

(b)

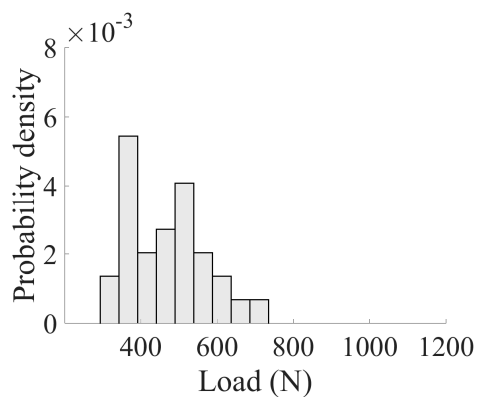

(c)

Figure 1. Probability density of force at failure for (a) small, (b) medium and (c) large specimens [6].

The failure loads can be transformed to the maximum tensile stresses at failure by

$$
\sigma_{f}=\frac{3 F_{f} L_{s}}{4 w h^{2}}
$$

where $F_{f}$ is the failure load, $L_{s}$ is the support span, $w$ is the width and $h$ is the thickness of the specimen. This leads to the range $\sigma_{f}=[110.8 ; 207.2]$, [86.4; 144.7] and [67.7; 153.8] MPa for the small, medium and large specimens, respectively. This implies that an increased area leads to a decrease in fracture strength. As an increased surface area would normally contain more flaws, this is an expected result.

\subsection{Quasi-Static Pressure Tests}

Quasi-static pressure tests were performed on 11 glass plates with dimensions of $400 \mathrm{~mm} \times 400$ $\mathrm{mm} \times 3.8 \mathrm{~mm}[6]$. The tests were carried out in a steel chamber, and the pressure loading was introduced by slowly filling the chamber with water. To enable the use of point tracking, two AVT Prosilica GC2450 high-speed cameras (with a logging rate of $15 \mathrm{~Hz}$ ) were used to record the tests. The glass plate was fixed between steel plates by 16 equidistant M6 bolts. To avoid premature damage of the glass, $4 \mathrm{~mm}$ thick rubber strips were placed on either side of it. Furthermore, to fasten the upper steel plate properly, while limiting the pressure on the rubber and glass, $11.7 \mathrm{~mm}$ thick steel stoppers were placed on the bolts. See Figure 2a for an illustration. Figure $2 b$ presents the applied pressure at failure. By analysing the problem in the finite element code Abaqus [10], without considering failure, the corresponding maximum in-plane stress was found to be in the range $\sigma_{f} \approx[54 ; 94] \mathrm{MPa}$.

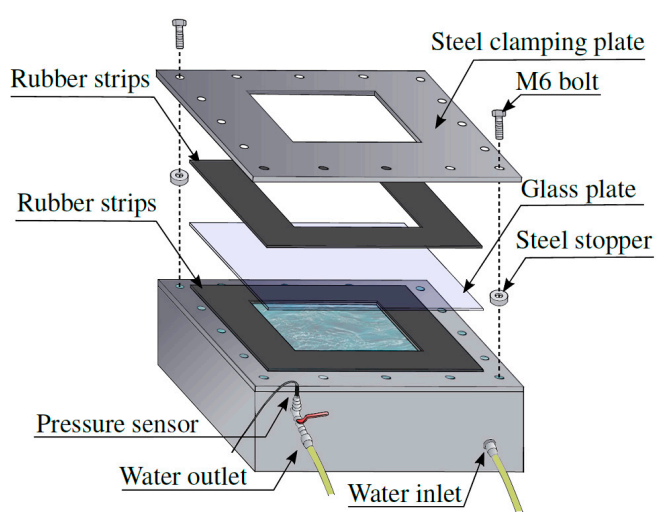

(a)

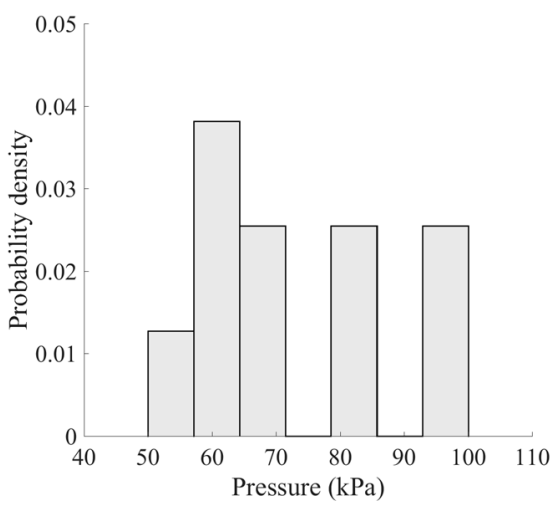

(b)

Figure 2. Quasi-static pressure tests: (a) assembly of the test set-up and (b) applied pressures at failure. 


\subsection{Shock Tube Tests}

Twelve blast pressure tests were performed on glass plates with dimensions of $400 \mathrm{~mm} \times 400$ $\mathrm{mm} \times 3.8 \mathrm{~mm}[6]$. The fastening of the glass plate in the SSTF was similar to the quasi-static pressure tests, with use of rubber strips and steel stoppers. Table 1 summarizes the results from these tests, where $P_{\max }$ (in $\mathrm{kPa}$ ) refers to the maximum overpressure, $t_{\text {frac }}$ (in $\mathrm{ms}$ ) is the time of fracture initiation, initiation, $D_{\text {max }}$ (in $\mathrm{mm}$ ) is the maximum centre displacement before fracture, and $v_{\text {frag }}$ (in $\mathrm{m} / \mathrm{s}$ ) is the maximum glass fragment velocity. The tests are divided into three groups (A, B, C), depending on $P_{\max }$.

Table 1. Summary of the blast tests in the SSTF [6].

\begin{tabular}{cccccccccc}
\hline Test & $\boldsymbol{P}_{\text {max }}$ & $\boldsymbol{t}_{\text {frac }}{ }^{\mathbf{1}}$ & $\boldsymbol{D}_{\text {max }}$ & $\boldsymbol{v}_{\text {frag }}$ & Test & $\boldsymbol{P}_{\text {max }}$ & $\boldsymbol{t}_{\text {frac }}{ }$ & $\boldsymbol{D}_{\text {max }}$ & $\boldsymbol{v}_{\text {frag }}$ \\
\hline A-01 & 53.0 & 1.21 & 4.52 & 15.8 & C-01 & 73.4 & 1.29 & 5.89 & 21.5 \\
A-02 & 51.6 & 1.38 & 5.06 & 14.7 & C-02 & 73.2 & 1.33 & 6.48 & 22.3 \\
\hline B-01 & 63.7 & 0.88 & 3.23 & 24.5 & B-05 & 63.3 & 1.00 & 4.29 & 21.8 \\
B-02 & 64.6 & 1.25 & 5.40 & 18.8 & B-06 & 62.9 & 0.92 & 3.68 & 19.3 \\
B-03 & 65.5 & 1.46 & 5.64 & 17.3 & B-07 & 64.0 & 1.33 & 5.77 & 19.0 \\
B-04 & 62.5 & $\times$ & 5.44 & $\times$ & B-08 & 62.9 & $\times$ & 5.96 & $\times$ \\
\hline
\end{tabular}

${ }^{1} t=0$ corresponds to the arrival time of the blast wave at the glass plate.

It can be seen from Table 1 that two out of the 12 glass plates did not fracture (B-04 and B-08). As the maximum applied pressure in e.g., group A was smaller, this illustrates the stochastic behaviour of glass fracture. Additionally, it can be seen that glass plates exposed to (almost) the same pressure, did not fracture at the same point in time.

\section{Strength Prediction Modelling}

A model for predicting the fracture strength of glass was recently proposed by Yankelevsky [7]. The model presented here is based on that work, but it includes additional features and adjustments.

\subsection{Model Basis and Procedure}

The basis of the strength prediction model is the fracture criterion given by Equation (1). For a given problem, the stresses in a glass plate (excluding fracture) can be provided by a finite element simulation, or if possible, an analytical calculation. In the strength prediction model, this information is combined with a flaw map, i.e., information about the position, size and orientation of the flaws in the glass plate. The flaw map is varied over $n$ iterations in a Monte Carlo simulation, and in each iteration, the fracture criterion given by Equation (1) is checked. From this, we can obtain $n$ values of the fracture strength (given by stress, load, displacement, etc.), and time and position of fracture initiation. An estimated failure percentage can also be found. See [6] for more details.

The required input of the strength prediction model is given below.

- $\quad$ Fracture toughness $K_{I C}$

- Flaw shape $a / c$
- $\quad$ Flaw density $\rho_{\max }$

- Maximum flaw depth $a_{\max }$ $a_{\max }$
- $\quad$ Area of jumbo plate $A_{\text {jumbo }}$

- Number of iterations $n$

- Stress history in glass from a FE simulation

The parameter $A_{\text {jumbo }}$ refers to the area of a larger glass plate (jumbo plate) in which the analysed specimens are cut from.

\subsection{Model Results}

In the following, all three presented experimental set-ups were analysed by the strength prediction model. The stress histories in the glass were obtained by simulations in Abaqus, which were validated by the experimental data (pre-fracture). The following input parameters were 
employed for the strength prediction analyses. It is referred to Osnes et al. [6] for a justification of the chosen parameters.

- $\quad K_{I C}=0.75 \mathrm{MPa} \sqrt{\mathrm{m}}[11]$

- $a / c=1$
- $\quad \rho_{\max }=2$ flaws $/ \mathrm{cm}^{2}$

- $a_{\max }=100 \mu \mathrm{m}$
- $A_{\text {jumbo }}=3210 \times 6000 \mathrm{~mm}^{2}$

- $n=5000$

In Figure 3, the applied load at fracture from the experiments and the strength prediction model is compared for the four-point bending tests and the quasi-static pressure tests in "box plots". In such a plot, the lower and the upper edges of the "box" mark the 25th and the 75th percentiles of the data, while the central line marks the median. The outer dashed lines include the rest of the data. For the four-point bending tests, the experimental results appeared within the predicted ones, with the median at approximately the same value. For the quasi-static pressure tests, the strength prediction model slightly overestimated the failure load; however, the majority of the test data (i.e., the "box") appeared within the predicted values. This deviation might be explained by subcritical crack growth, i.e., stable growth of the flaws before failure. This effect is not considered in the model.

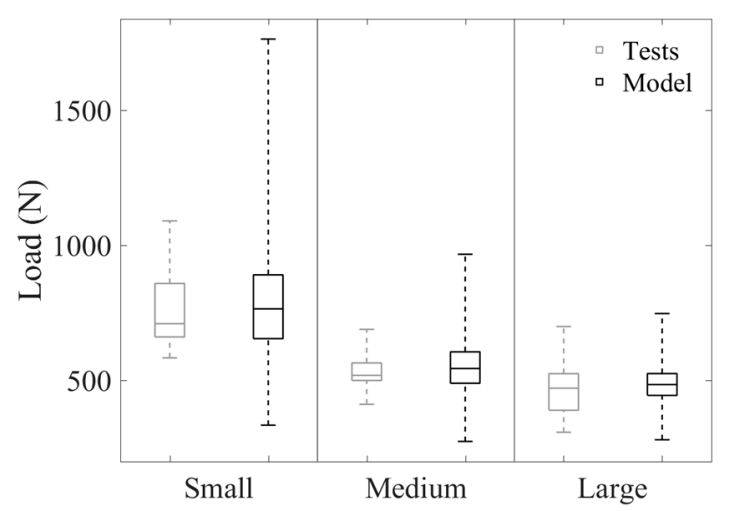

(a)

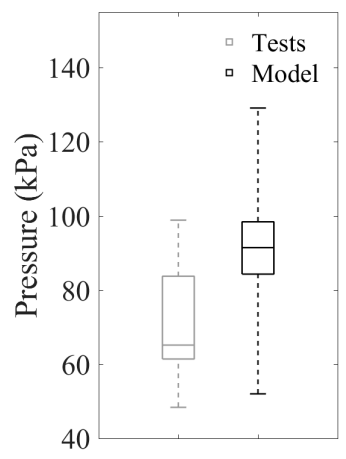

(b)

Figure 3. Failure load determined by laboratory tests and the strength prediction model in (a) fourpoint bending tests and (b) quasi-static pressure tests.

For the shock tube tests, the load was not gradually applied as for the quasi-static tests, and a failure load could not be compared for the tests and model in the same manner. Instead, the time of fracture initiation $t_{\text {frac }}$ is presented (see Figure 4a). Additionally, the predicted failure percentage was found to be $94.2,99.8$ and 100 for group A, B and C, respectively. Compared to the experimental results, this is too high. However, it should be noted that to draw any definite conclusions, a much larger number of experimental tests must be carried out. Nevertheless, it is known that the fracture strength of glass generally increases with the loading rate [4], and the chosen value for $K_{I C}$ might not be applicable. Therefore, a new simulation with an increased value $(0.85 \mathrm{MPa} \sqrt{\mathrm{m}})$ was performed (see Figure $4 b$ ).

The failure percentages were found to be 53.4, 94.6 and 100 for group A, B and C, which agrees better with the experimental results. The median values for $t_{\text {frac }}$ found in the experiments are also closer to the predicted ones. Note that $K_{I C}$ should in reality depend on the strain rate rather than being kept constant. 


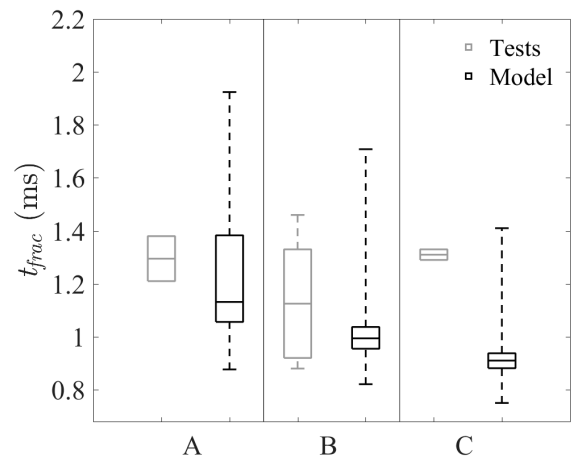

(a)

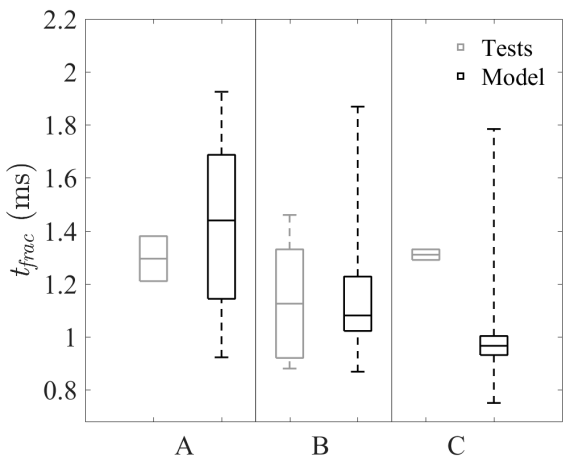

(b)

Figure 4. Time of fracture initiation $t_{\text {frac }}$ in shock tube tests for group A, B and C determined by experiments and the strength prediction model, with $K_{I C}$ set to (a) $0.75 \mathrm{MPa} \sqrt{\mathrm{m}}$ and (b) $0.86 \mathrm{MPa} \sqrt{\mathrm{m}}$ in the model.

\section{Concluding Remarks}

The fracture strength of glass has been investigated in three different experimental set-ups; quasi-static four-point bending tests, quasi-static pressure tests and dynamic blast pressure tests. As expected, the experiments illustrated the stochastic nature of fracture in glass. This was shown by large variations in fracture strength within the same test set-up, including dependency on the loading and boundary conditions. Furthermore, a strength prediction model for glass, which is based on the existence of microscopic surface flaws, has been applied. It was shown that the model has a great potential when it comes to predicting the fracture strength in glass, as it managed to successfully capture many trends in the experiments. However, to improve the performance and accuracy of the model, a more thorough investigation of both glass' loading rate dependency and the input parameters is necessary.

Author Contributions: The presented study has been conducted by K.O. in connection with her Ph.D. studies at NTNU, under supervision of T.B. and O.S.H.

Acknowledgments: The authors gratefully appreciate the financial support from CASA (project number 237885), Centre for Research-based Innovation (CRI), at the Norwegian University of Science and Technology

Conflicts of Interest: The authors declare no conflict of interest.

\section{References}

1. Shariat, S.; Mallonee, S.; Stephens-Stidham, S. Summary of Reportable Injuries in Oklahoma: Oklahoma City Bombing Injuries. 1998. Available online: https://www.ok.gov/health2/documents/OKC_Bombing.pdf (accessed on 13 July 2018).

2. Beason, W.L.; Morgan, J.R. Glass failure prediction model. J. Struct. Eng. 1984, 110, 197-212.

3. Wachtman, J.B.; Cannon, W.R.; Matthewson, M.J. Mechanical Properties of Ceramics, 2nd ed.; John Wiley \& Sons: Hoboken, NJ, USA, 2009.

4. Larcher, M.; Solomos, G.; Casadei, F.; Gebbeken, N. Experimental and numerical investigations of laminated glass subjected to blast loading. Int. J. Impact Eng. 2012, 39, 42-50.

5. Aune, V.; Fagerholt, E.; Langseth, M.; Børvik, T. A shock tube facility to generate blast loading on structures. Int. J. Prot. Struct. 2016, 7, 340-366.

6. Osnes, K.; Børvik, T.; Hopperstad, O.S. Shock tube testing and strength modelling of annealed float glass. 2018, in press.

7. Yankelevsky, D.Z. Strength prediction of annealed glass plates-A new model. Eng. Struct. 2014, 79, 244255.

8. Newman, J.; Raju, I. An empirical stress-intensity factor equation for the surface crack. Eng. Fract. Mech.1981, 15, 185-192. 
9. Fagerholt, E. Field Measurements in Mechanical Testing Using Close-Range Photogrammetry and Digital Image Analysis. Ph.D. Thesis, Norwegian University of Science and Technology (NTNU), Trondheim, Norway, 2012.

10. Dassault Systemes Simulia Corporation. Abaqus, Version 6.14; Dassault Systemes Simulia Corporation: Providence, RI, USA, 2014.

11. Anstis, G.; Chantikul, P.; Lawn, B.R.; Marshall, D. A critical evaluation of indentation techniques for measuring fracture toughness: I, Direct crack measurements. J. Am. Ceram. Soc. 1981, 64, 533-538.

(C) 2018 by the authors. Licensee MDPI, Basel, Switzerland. This article is an open access article distributed under the terms and conditions of the Creative Commons Attribution (CC BY) license (http://creativecommons.org/licenses/by/4.0/). 